\title{
A Multicriteria Intelligence Aid Methodology Using MCDA, Artificial Intelligence, and Fuzzy Sets Theory
}

\begin{abstract}
Anissa Frini
Sciences de la Gestion, Université du Québec à Rimouski, Campus de Lévis, Lévis, QC, Canada

Correspondence should be addressed to Anissa Frini; anissa_frini@uqar.ca

Received 15 May 2017; Revised 1 September 2017; Accepted 11 September 2017; Published 31 October 2017

Academic Editor: Juan C. Leyva

Copyright (C) 2017 Anissa Frini. This is an open access article distributed under the Creative Commons Attribution License, which permits unrestricted use, distribution, and reproduction in any medium, provided the original work is properly cited.

Intelligence is increasingly relevant today in both military and business intelligence contexts. Business executives, military, and governments have more large datasets and meet difficulties in anticipating threat/competitor future decisions. Decision anticipation is desirable because it will enhance situation understanding and then will limit the surprise effect and favor more appropriate reactions and decision-making. Generating and evaluating competitor/threat actions is a very challenging problem because of the uncertainty, incompleteness, and ambiguity associated with it. This paper extends the multicriteria decision aid (MCDA) methodology to the context of intelligence analysis and proposes the main pillars of a novel methodology called "Multicriteria Intelligence Aid" (MCIA). More specifically, this paper addresses how can we adapt MCDA to the context of intelligence analysis and how can we use existent methods and techniques from MCDA, artificial intelligence, and fuzzy sets theory to build this methodology. The paper presents the MCIA steps, which consist of (i) structuring the competitor/threat decision problem, (ii) handling imperfect data, (iii) modeling the analyst's risk attitude, and (iv) aggregating the performance of the generated potential actions. An illustration of the methodology is provided in the military context. Results show that the novel methodology is applicable and provides interesting and valuable results.
\end{abstract}

\section{Introduction}

In the sciences of operations research and decision theory, the multicriteria decision aid methodology has seen considerable development, leading to a great variety of methods belonging either to the single-criterion synthetizing approach or to the outranking approach. While the former approach builds an aggregation function using the individual scores with regard to each criterion in order to represent a global score for each alternative, the latter is inspired from the social choice theory and is based on the pairwise comparison of actions. Both approaches are used to support complex decisions, where multiple, conflicting, and incommensurate points of view (objectives, criteria/attributes, etc.) need to be considered simultaneously in the decision-making process. The multicriteria decision aid (MCDA) methodology has proven, along many years of practice, its relevance, utility, and value added to decision theory. It assumes a dialogue with the decision-maker and aims at recommending the best compromise solution based on the decision-maker's preferences. The multicriteria decision aid process is a recursive and nonlinear process composed of the following steps: structuring of the decision-problem, local preference modeling, aggregation, exploitation, and recommendation. The MCDA process involves both the decision-maker and the analyst who supports him. But what could happen if the decision-maker is not involved in the multicriteria decision aid process? Would the MCDA methodology continue to be valuable? This is specifically the question we are dealing with in this paper. We consider more specifically the context of anticipation of decisions in the intelligence domain (e.g., business intelligence or military intelligence). The objective of the paper is to propose a methodology that supports the intelligence analyst in his duty and more specifically in determining the adversary (competitor/threat) future decisions. Here, the decision-maker (the competitor or the threat) and his preferences are unknown and no dialogue could take place. Consequently, we cannot, as in the classical MCDA, 
structure the problem along with the decision-maker, but rather we need to use available data to predict the decisionmaker's preferences and anticipate his future decisions.

The topic of intelligence support addressed in this paper is increasingly relevant today in both military and business intelligence contexts. In fact, the generation of threat/competitor potential future decisions and their ranking will enhance the situation understanding and then will limit the surprise effect and favor more appropriate reactions. However, generating and evaluating competitor/threat actions is a very challenging problem because of the great amount of uncertainty, incompleteness, and ambiguity associated with it.

In this paper, we start from the belief that the multicriteria methodology, when used conjointly with artificial intelligence (AI) techniques, is relevant and of interest as a tool for the intelligence analyst. The general objective of this paper is to extend the multicriteria decision aid methodology to the context of intelligence. The main pillars of a new methodology called "Multicriteria Intelligence Aid" will be proposed in this paper. Table 1 presents the differences between the decision-making problem for which MCDA is valuable and the intelligence problem for which we presume MCIA will be a valuable approach.

Two distinct but related research questions will be addressed in this paper in order to shed some light on this topic:

(i) How can we exploit and adapt multicriteria decision support approaches to the context of intelligence analysis?

(ii) Are existent methods and techniques from MCDA, artificial intelligence, and fuzzy sets theory sufficient to build the novel MCIA methodology?

To answer these research questions, this paper proposes a multidisciplinary methodology using a panoply of techniques from artificial intelligence, MCDA, and fuzzy multicriteria decision analysis to support intelligence analysts in anticipating the more likely potential adversary actions.

This paper will be structured as follows. Section 2 will review previous works. Then, Section 3 will present the Multicriteria Intelligence Aid methodology. All MCIA steps from the structuring phase to the recommendation will be discussed. Finally, Section 4 will illustrate the proposed methodology in the military intelligence context.

\section{Previous Works}

The review of the literature shows that there are at least two main approaches to deal with competitor or military intelligence: the red teaming and the game theory. Red teaming (RT) is used for military intelligence and planning. It consists of a simulation of blue and red teams, where a blue team represents the intent, objectives, and interests of the friendly force and a red team emulates enemies and reproduces their motivations, intentions, behaviours, and anticipated actions. Computational red teaming (CRT) is concerned with the computational side of RT in order to augment a human-based RT exercise with computational models and methods. Abbass et al. [1] define five different levels of red team modeling. These levels represent increasing degrees of red team adaptability and system complexity. CRT0 equips agents with a generic decision-making model. CRT1 introduces learning (social and evolutionary), in the sense of the agent's ability to change its decision-making process. CRT2 level of red modeling consists of evolutionary algorithms searched for communication strategies that would give the blue team a competitive advantage over a fixed red team. When system-level adaptation occurs within an environment that itself changes and evolves, then we have a red team adaptation at level CRT3. The highest level CRT4 provides the full potential of "learning about competitors" by adding mechanisms of reflection [1].

On another side, game theory is a rigorous framework for explaining and anticipating competitor behaviours. This well-known theory could appear unmanageable in the real word. Coyne and Horne [2] assert that "game theory models become unwieldy when a competitor has many options, when the strategist is unsure which metrics his rival will use to evaluate them or when there are multiple competitors, each of whom might react differently." In this paper, we are interested in those specific situations when a competitor has many options and we are uncertain about the metrics he will be using and the ratings he will be giving to each potential action. For these specific problems, many points of view (objectives, criteria, etc.) could enter into consideration in order to best predict the red team's decisions. Multiobjective evolutionary computation and multicriteria decision analysis (MCDA) are interesting computational approaches that could be of interest for this topic. Multiobjective computation consists of solving decision-making problems under multiple objectives (to be optimized), where the set of solutions is defined by a set of constraints. The MCDA approaches are discrete approaches that consider decision-making problems, where a set of discrete alternatives are predefined and the decision-maker has to choose among these alternatives.

Multiobjective evolutionary computation is a set of techniques capable of searching for the set of solutions that represent the nondominated solutions considering the competing objectives. In the last decade, the field of multiobjective evolutionary computation has grown with significant number of efficient algorithms [3-7]. Miettinen [8] categorizes the main approaches in the multiobjective optimization literature into four categories. The first approach does not use preference information and is called no-preference. The second approach finds all possible nondominated solutions and then applies the user's preferences (posterior approach). The third approach incorporates user preference prior to the optimization process (a priori approach). The fourth approach combines the posterior and a priori approaches (interactive approach).

In the discrete case when the alternatives are predefined, two main MCDA approaches are developed: the single-criterion approach and the outranking synthesising approach. The single-criterion approach consists of building an aggregation function using the individual scores with regard to each criterion in order to represent the global score 
TABLE 1: MCDA versus MCIA.

\begin{tabular}{|c|c|c|}
\hline & MCDA & MCIA \\
\hline Characteristics of the problem & $\begin{array}{l}\text { Decision-aid problems: complex, ill-defined, and } \\
\text { human-oriented decision problems. }\end{array}$ & $\begin{array}{l}\text { Intelligence support: complex, ill-defined, } \\
\text { and human-oriented decision problems, } \\
\text { uncertainty, need for anticipation, inference, } \\
\text { and prediction. }\end{array}$ \\
\hline Decision-maker & Involved in the process. & $\begin{array}{l}\text { Competitor/threat; not involved in the } \\
\text { process. }\end{array}$ \\
\hline Alternatives/actions & $\begin{array}{l}\text { Could be generated with techniques and tools for } \\
\text { stimulation of creativity. The decision-maker } \\
\text { generally participates in the action generation step. }\end{array}$ & $\begin{array}{l}\text { Potential actions which need to be generated } \\
\text { and anticipated from the } \\
\text { competitor's/threat's available data on his } \\
\text { present and past activities. }\end{array}$ \\
\hline Criteria & $\begin{array}{l}\text { Defined with top-down approach and bottom-up } \\
\text { approaches. }\end{array}$ & $\begin{array}{l}\text { Defined with top-down approach and } \\
\text { bottom-up approaches and assumes that the } \\
\text { competitor/threat objectives are well known. }\end{array}$ \\
\hline Ratings & Crisp or imperfect. & $\begin{array}{l}\text { Imperfect: imprecise, uncertain, ambiguous, } \\
\text { and subjective. }\end{array}$ \\
\hline Preferences & $\begin{array}{l}\text { Elucidated and modeled by an interactive process } \\
\text { with the decision-maker. }\end{array}$ & $\begin{array}{l}\text { Need to be inferred from available data } \\
\text { using artificial intelligence techniques. }\end{array}$ \\
\hline Methodology objective & $\begin{array}{l}\text { Screening, prioritizing, ranking, or selecting a set of } \\
\text { decision-maker actions under independent, } \\
\text { incommensurate, or conflicting criteria. }\end{array}$ & $\begin{array}{l}\text { Generating and prioritizing a set of } \\
\text { competitor/threat potential actions under } \\
\text { independent, incommensurate, or } \\
\text { conflicting criteria. }\end{array}$ \\
\hline
\end{tabular}

of each alternative. The outranking synthesising approach is inspired from the social choice theory. It is based on the pairwise comparison of the COAs along each criterion. Each criterion is considered as a voter, with a particular voting power fixed accordingly with the analyst, and each COA as a candidate [9]. Most MCDA methods assume that the ratings of alternatives and the weights of criteria are crisp numbers. However, this is not always realistic because uncertainties could arise from information that is not quantifiable, incomplete, or difficult to obtain because of partial ignorance. The review of the literature shows that probabilistic methods were explored in dealing with MCDA under uncertainty $[10,11]$ as well as fuzzy set theory [12-15]. More specifically, for fuzzy multicriteria analysis, there exist four different methodologies: the fuzzy ranking methods, the fuzzy analytic hierarchy process (AHP) methods, defuzzification based methods, and fuzzy outranking methods. Some examples of fuzzy methods have been developed in Yager [15], Yager [16], Chen [17], Deng [18], Cheng et al. [19], Yeh and Deng [20], and Ribeiro [14]. Fuzzy methods were derived from the approximation approach as the fuzzy $\max -\min [21]$ and the fuzzy weighted sum [22-24]. Other researches also generalized some classical methods to the fuzzy context such as fuzzy TOPSIS [17] and fuzzy AHP [19].

Both multiobjective evolutionary computation and MCDA are decision-making methods that could not be used "as is" for intelligence. Their methodologies need to be adapted to the intelligence context.

Extending MCDA to the context of intelligence is the main idea advanced in this paper. To the best of our knowledge, the literature review reveals only one master thesis on the effectiveness of multicriteria intelligence matrices for intelligence [25]. This research work discusses relevant literature existing on the conventional form of MCDA and addresses the difference necessary to convert MCDA to an intelligence methodology. It did not propose a new methodology but rather tested empirically the value of using multicriteria intelligence matrices to support analysts in their intelligence work. More specifically, the author conducted controlled experiment with intelligence analysts to test the hypothesis that MCDA is a valuable method to use in intelligence. Initial findings suggest that multicriteria intelligence matrices are likely to be a valuable method to use when conducting intelligence analysis. As there is no work on the methodological side to convert MCDA to a new methodology valuable for intelligence context, this paper will propose the main pillar of a new MCIA methodology. To develop this methodology, a combination of multidisciplinary existing methods from MCDA, artificial intelligence, and fuzzy sets theory is proposed.

\section{A Multicriteria Intelligence Aid (MCIA) Methodology}

In this section, we present the main steps of the proposed Multicriteria Intelligence Aid (MCIA) methodology. The proposed methodology is presented in general terms and could be applied in the context of either competitor intelligence or military intelligence. The terms "adversary" and "competitor/threat" will be used and will refer to the competitor if in business context or the threat if in military context. The MCIA methodology proposed here aims at supporting intelligence analyst in structuring the adversary decision-making problem and anticipating threats and their actions. All MCIA steps from the structuring phase to the recommendation will be highlighted. 
Mainly, the methodology is composed of the following five steps as illustrated in Figure 1.

Step 1. Structuring the competitor/threat decision-making problem:

(i) Generating threats.

(ii) Anticipating adversary potential actions.

(iii) Defining the list of criteria and evaluating the potential actions according to these criteria.

(iv) Modeling the competitor/threat preferences.

Step 2. Handling imperfect data.

Step 3. Modeling the analyst's risk attitude.

Step 4. Multicriteria performance aggregation.

In order to detail all the steps of the methodology presented in Figure 1, a multidisciplinary approach is needed. Techniques and tools from multicriteria decision aid, artificial intelligence, uncertainty modeling, and fuzzy sets will be combined together. In this paper, we focus on the following steps: threat and action generation, handling uncertainty and imprecision, modeling the analyst's risk attitude, and the performance aggregation. We show how existent techniques and methods in MCDA, AI, and fuzzy sets could contribute to the development of such a methodology. This paper supposes that the adversary preferences are known from available data and modeled. Development in this area is out of the scope of this paper.

\subsection{Step 1: Structuring the Competitor/Threat Decision-Making} Problem. Structuring the competitor/threat decision-making problem consists of four activities: generating threats, anticipating competitor/threat potential actions, defining the list of criteria, and evaluating the actions according to this set of criteria.

(a) Generating Competitor/Threat Potential Actions. In practice, a panoply of techniques and tools for stimulation of creativity are used for actions generation. All these techniques suppose that the decision-maker is known and could participate in action generation process. In intelligence context (for either business intelligence or military intelligence), this is not the case, since the decision-maker is not involved in the process. The analysis of information from multiple heterogeneous sources supports the analyst in the generation of threats and the anticipation of actions. Though under time pressure and data overload, the analyst needs to be supported in this effort because of his limited ability to rigorously follow effective methods for generating, managing, and evaluating hypothesis. In such situations, we cannot, as in classical MCDA, structure the problem along with the decisionmaker, but rather we need to use historical/available data to anticipate his future decisions. AI techniques and specifically reasoning techniques are valuable techniques to address action generation in MCIA context. (b) Defining the List of Criteria and Evaluating the Actions according to These Criteria. In order to prioritize the potential actions inferred in the previous step, the intelligence analyst will need first to define the list of criteria the threat is more likely to consider. The top-down approach of Keeney [26] and the bottom-up approach of Roy [9] are still valuable in intelligence analysis context. The top-down approach derives the criteria by decomposing the objectives and the bottomup approach develops the criteria by analyzing and regrouping the action's consequences. In particular, in intelligence context and with Kenney's approach, it is important for the intelligence analyst to have a clear understanding of the adversary general and specific objectives.

Once the list of criteria is defined, the intelligence analyst has to evaluate how well each competitor/threat potential action meets each criterion. Classical MCDA methods suppose that the ratings of alternatives are crisp numbers. In intelligence context, this is recognized as unrealistic and it is unlikely that the analyst will be able to give such crisp ratings. He will be necessarily imprecise. Thus, to overcome this difficulty, it is more natural for the analyst, when evaluating the competitor/threat actions, to describe the action's ratings according to each criterion in linguistic terms, for example, "very low," "medium," "high," and "fair." Suppose that we have $m$ actions $A_{i}(i=1, \ldots, m)$ and $n$ criteria $C_{j}(j=$ $1, \ldots, n)$. The evaluation matrix is expressed by

$$
A_{i}\left[\begin{array}{ccc}
\tilde{x}_{11} & \cdots & \tilde{x}_{1 n} \\
\vdots & \ddots & \vdots \\
\tilde{x}_{m 1} & \cdots & \tilde{x}_{m n}
\end{array}\right],
$$

where $\tilde{x}_{i j}$ represents the linguistic rating of alternative $A_{i}$ with respect to criterion $C_{j}$. The relative importance of criteria is given by $\widetilde{W}=\left(\widetilde{w}_{i}\right)$, where $\widetilde{w}_{j}$ represents the weight of criterion $C_{j}$.

In MCDA, such matrices are called "decision matrices," but we prefer to use here the term "intelligence matrices" because they will be used in the perspective of intelligence support instead of decision support.

Once the multicriteria intelligence matrix is completed with the linguistic variables, the next step will consist of adversary preference modeling. In classical MCDA, the preference elicitation could be done using a panoply of techniques: utility theory, valued functions, pairwise comparison, tradeoffs, discrimination thresholds, and so forth. However, in the context of intelligence underlying MCIA, these techniques need to be adapted and combined with reasoning techniques to be able to infer the preferences from past adversary reactions. AI techniques like case-based reasoning while combined with pairwise comparison and tradeoffs will be valuable to answer this challenging question.

3.2. Step 2: Handling Imperfect Data. The MCIA methodology has to deal with imperfect data. Imperfect data includes data which is uncertain, imprecise, or ambiguous. This imperfection is inherent to the intelligence context and cannot 


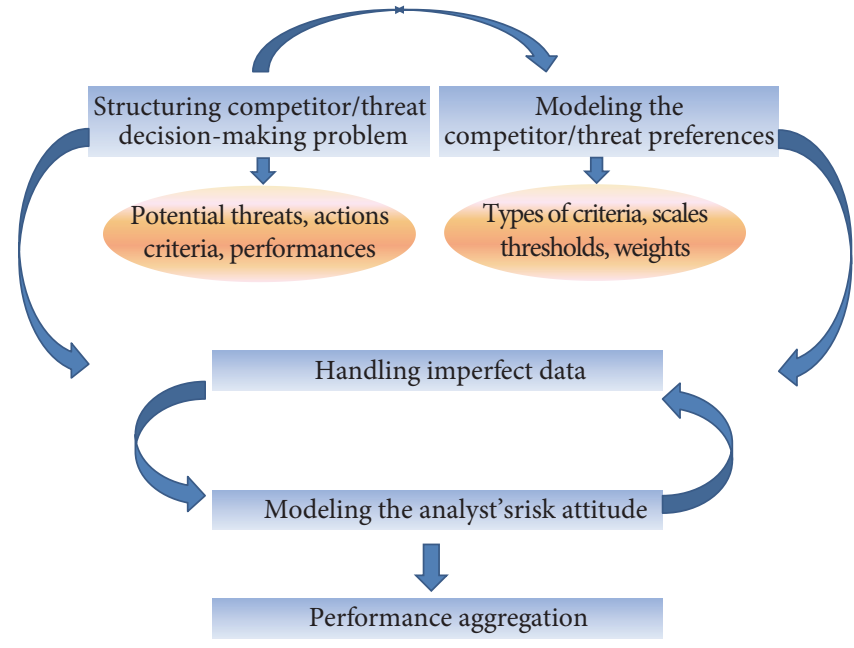

Figure 1: MCIA methodology.

be ignored, specifically the imprecision of the ratings which is a common problem for intelligence situations. In fact, it is difficult for the analyst to predict precise ratings of the adversary actions according to the list of criteria as if the competitor/threat would have done. However, imprecision is not the only imperfection that needs to be considered. In fact, many types of uncertainty and risk exist. Stewart [28] defines two categories of uncertainty: external uncertainty and internal uncertainty. External uncertainty refers to the lack of knowledge about the consequences of a particular choice (decision). This includes (i) random uncertainty that arises because of unpredictable state of the nature (this is outside the control of the analyst) and (ii) epistemic uncertainty that is due to lack of data and knowledge about different phenomena. Internal uncertainty is related to ambiguity/imprecision of the data because it refers to human judgments, preferences, values, and risk attitudes.

The MCIA methodology proposed in this paper models internal uncertainty due to imprecision and subjective human judgment. As discussed earlier, because of the difficulty encountered by the analyst while evaluating competitor/threat actions, the ratings of the actions will be expressed in linguistic terms, for example, "very low," "medium," "high," and "fair." Then, the linguistic decision analysis approach [29-31] will be used as an approximate way to represent natural words or sentences used in human judgment and perception. Accordingly, the linguistic description of the analyst will be transformed into fuzzy numbers in order to deal with the uncertainty of the ratings. A fuzzy number is a convex fuzzy set, characterized by a given interval of real numbers, each with a grade of membership between 0 and 1 . At this step, the analyst will make the choice of a type of fuzzy numbers (triangular, trapezoidal, etc.) depending on how he will perceive the imprecision related to each criterion.

If the analyst chooses triangular fuzzy numbers, the membership function will be defined as follows:

$$
\mu_{\widehat{a}}(x)= \begin{cases}\frac{\left(x-a_{1}\right)}{\left(a_{2}-a_{1}\right)}, & a_{1} \leq x \leq a_{2}, \\ \frac{\left(a_{3}-x\right)}{\left(a_{3}-a_{2}\right)}, & a_{2} \leq x \leq a_{3}, \\ 0, & \text { otherwise. }\end{cases}
$$

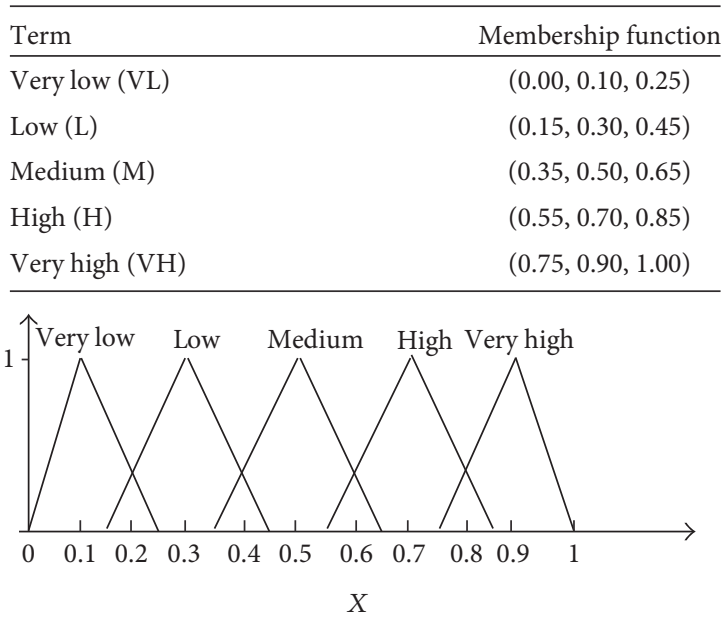

FIGURE 2: Fuzzy triangular membership functions.

The triangular fuzzy number is based on a three-value judgment: the minimum possible value $a_{1}$, the most possible value $a_{2}$, and the maximum possible value $a_{3}$. Example of linguistic variables modeled with fuzzy numbers is given in Figure 2.

3.3. Step 3: Modeling the Analyst's Risk Attitude. When evaluating and ranking different competitor's/threat's actions, the intelligence analyst could express different risk attitudes (from absolutely optimistic to absolutely pessimistic), depending on the context, on the significance of the action, and on its consequences. The attitude towards risk of the analyst will have an impact on the ranking of the different actions. The intelligence analyst's risk attitude could also differ from one criterion to another depending on the relative importance of each criterion. Ultimately, the analyst would not like to take risk for the evaluation of the criteria with high relative importance. Analyst's risk attitude is a valuable way to tackle not only the imprecision but also the subjectivity related to his judgments. 
TABLE 2: Linguistic terms of risk attitudes and their related fuzzy numbers [27].

\begin{tabular}{lcc}
\hline Linguistic term & $\begin{array}{c}\text { Triangular fuzzy number } \\
\text { derived from }\left(a_{1}, a_{2}, a_{3}\right) \text { for } \\
\text { criteria to be maximized }\end{array}$ & $\begin{array}{c}\text { Triangular fuzzy number } \\
\text { derived from }\left(a_{1}, a_{2}, a_{3}\right) \text { for } \\
\text { criteria to be minimized }\end{array}$ \\
\hline Absolutely optimistic (AO) & $\left(a_{1}, a_{3}, a_{3}\right)$ & $\left(a_{1}, a_{1}, a_{3}\right)$ \\
Very optimistic (VO) & $\left(a_{1},\left(a_{2}+3 a_{3}\right) / 4, a_{3}\right)$ & $\left(a_{1},\left(a_{2}+3 a_{1}\right) / 4, a_{3}\right)$ \\
Optimistic (O) & $\left(a_{1},\left(a_{2}+a_{3}\right) / 2, a_{3}\right)$ & $\left(a_{1},\left(a_{2}+a_{1}\right) / 2, a_{3}\right)$ \\
Fairly optimistic (FO) & $\left(a_{1},\left(3 a_{2}+a_{3}\right) / 4, a_{3}\right)$ & $\left(a_{1},\left(3 a_{2}+a_{1}\right) / 4, a_{3}\right)$ \\
Neutral & $\left(a_{1}, a_{2}, a_{3}\right)$ & $\left(a_{1}, a_{2}, a_{3}\right)$ \\
Fairly pessimistic (FP) & $\left(a_{1},\left(3 a_{2}+a_{1}\right) / 4, a_{3}\right)$ & $\left(a_{1},\left(3 a_{2}+a_{3}\right) / 4, a_{3}\right)$ \\
Pessimistic (P) & $\left(a_{1},\left(a_{2}+a_{1}\right) / 2, a_{3}\right)$ & $\left(a_{1},\left(a_{2}+a_{3}\right) / 2, a_{3}\right)$ \\
Very pessimistic (VP) & $\left(a_{1},\left(a_{2}+3 a_{1}\right) / 4, a_{3}\right)$ & $\left(a_{1},\left(a_{2}+3 a_{3}\right) / 4, a_{3}\right)$ \\
Absolutely pessimistic (AP) & $\left(a_{1}, a_{1}, a_{3}\right)$ & $\left(a_{1}, a_{3}, a_{3}\right)$
\end{tabular}

To model the risk attitude of the intelligence analyst, we consider the work of Fenton and Wang [27] which proposes how to incorporate the DM's risk attitudes into the general fuzzy MCDA approach. This attitude risk modeling is logical and interesting. We make the choice to use this model because it considers linguistic terms modeled by fuzzy sets as is the case in our context. In fact, intelligence analyst would only be able to express qualitatively (with linguistic terms) the evaluation because of the uncertainty, imprecision, and ambiguity associated with the context. The research work of Fenton and Wang [27] is proposed in the context of decision-making but could be simply adopted in the context of intelligence analysis support. The authors use natural language to describe an appropriate range of attitudes between the extremes of "optimism" and "pessimism." In Fenton and Wang [27], nine terms expressing nine different attitudes towards risk were considered based on Miller's theory of cognitive retention [32]. Then, for each term, the fuzzy number expressing the linguistic variable (ratings of the action on certain criteria) is modified to model the extent to which the evaluation needs to be impacted by the decision-maker's risk attitude.

To incorporate the risk attitude to the triangular fuzzy number $\left(a_{1}, a_{2}, a_{3}\right)$, Fenton and Wang [27] use an ordered structure. As shown in Table 2, actions evaluation will be modeled with $\left(a_{1}, a_{2}, a_{3}\right)$ for neutral attitude and with $\left(a_{1}\right.$, $\left.a_{3}, a_{3}\right)$ and $\left(a_{1}, a_{1}, a_{3}\right)$, respectively, for absolutely optimistic (AO) and absolutely pessimistic (AP) for criteria to be maximized. If the criteria have to be minimized, $\left(a_{1}, a_{3}, a_{3}\right)$ and $\left(a_{1}, a_{1}, a_{3}\right)$ will, respectively, model the fuzzy number for absolutely pessimistic (AP) and absolutely optimistic (AO).

In the MCIA methodology, the fuzzy ratings will be transformed using formulas in Table 2 in order to express the subjectivity of the intelligence analyst. Depending on the criteria weight, the intelligence analyst's attitude towards risk could differ significantly. He could, for instance, show neutral attitude if the criteria are neither important nor unimportant. In case the criteria are extremely important, the analyst could show pessimistic attitude and avoid being too optimistic for fear that in reality the competitor/threat will not rate the action so high. Figure 3 illustrates the impact of the risk attitude on the membership function.

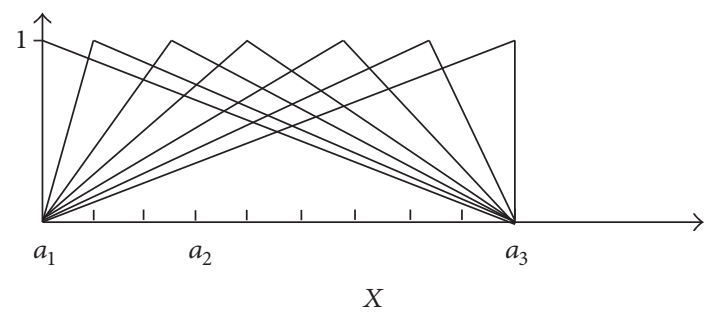

FIGURE 3: Transformation of the membership function depending on the analyst's risk attitude.

3.4. Step 4: Multicriteria Performance Aggregation. To perform the ranking of the different competitor/threat actions, we propose using a fuzzy MCDM method. As shown in Section 2, many fuzzy methods have been developed in the literature. Specifically, methods belonging to one of these four fuzzy methodologies could be considered: the fuzzy ranking methods, the fuzzy analytic hierarchy process (AHP) methods, defuzzification based methods, and fuzzy outranking methods. Each category has its advantages and disadvantages. As previously indicated in Section 2, a relatively great number of methods do exist. Some examples of fuzzy methods have been developed in Yager [15], Yager [16], Chen [17], Deng [18], Cheng et al. [19], Yeh and Deng [20], and Ribeiro [14]. Some fuzzy methods were derived from the approximation approach as the fuzzy max-min [21] and the fuzzy weighted sum [22-24]. Other researches also generalized some classical methods to the fuzzy context such as fuzzy TOPSIS [17] and fuzzy AHP [19]. We just need in this step of the MCIA methodology to be cautious and to make an informed choice for the method based on its strengths and weaknesses.

\section{Illustration}

In the next paragraphs, we present an illustration of the proposed methodology in the military context. Let us consider a military intelligence analyst facing a threat situation for which he is looking to anticipate threat future actions. 
TABLE 3: List of criteria.

\begin{tabular}{ll}
\hline Suitability & \\
C1 & Accomplishing threat's objectives (to be maximized) \\
C2 3 & Consistency with doctrine and past activities (to be maximized) \\
Taking advantage of adversary capability (to be maximized) & Taking advantage of the battlefield environment (to be maximized) \\
C4 & Taking advantage of the friendly disposition (to be maximized) \\
C5 & \\
Risk 65 & Personnel loss (to be minimized) \\
C7 & Collateral damage (to be minimized) \\
C8 & Confrontation risk (to be minimized) \\
C9 & Equipment reliability (to be maximized) \\
C10 & Personnel effectiveness (to be maximized) \\
Complexity & \\
C11 & Operation complexity (to be minimized) \\
C12 & Logistic complexity (to be minimized) \\
Loss of opportunities & \\
C13 & Cost of resources (to be minimized) \\
\hline
\end{tabular}

\subsection{Step 1: Structuring the Threat Decision-Making Problem}

(a) Generating Threat Potential Actions. In this step, reasoning techniques could be combined together and with the intelligence analyst's inputs in order to efficiently generate the threats and their actions. This is an interactive action generation method that is implemented within a semiautomated system. With an appropriate graphical user interface (GUI), the analyst is able to specify all the information that is relevant for the given situation and the uncertainty about the situation. From this original representation, the user will already be able to identify potential threats and specify possible courses of action (COA) for them. Then, the situation representation will be transformed into a situation model. A situation model is a formal representation of a situation, its dependencies, and its uncertainty. Afterwards, situational facts will be extracted from the situation model and various automated reasoning approaches (rule-based, description logics, and case-based reasoning) will infer new situational facts from existing ones. The inferred situational facts will be used to augment the initial situation model. In this illustration, case-based reasoning (CBR) will be used for this inference. CBR allows identifying past situations that are similar to the current situation. This is done using a similarity measure. A similarity measure takes into account and weighs every situational fact present in the situation template. It compares them with the situational facts present in past situations. Each identified past situation will have a set of identified threats and COAs. The user will analyze them and identify threats and COAs relevant to his context. For the sake of illustration, we consider that the intelligence analyst has inferred four potential actions $(A 1, A 2, A 3$, and $A 4)$.

(b) Defining the List of Criteria and Evaluating the Actions according to These Criteria. Table 3 presents a list of criteria which is applied in this context to evaluate the threat potential actions. Four criteria (suitability, risk, complexity, and loss of opportunity) are considered with a set of indicators ( $C 1$ to C13) allowing measuring each one of them.

Once the list of criteria is defined, the intelligence analyst has to evaluate how well each competitor/threat potential action meets each criterion. It is more natural for the analyst, when evaluating the competitor/threat" actions, to describe the action's ratings in linguistic terms, for example, "very low," "medium," "high," and "fair." The intelligence analyst will complete a linguistic evaluation of the potential actions according to suitability, complexity, risk, and loss of opportunities/cost. It is assumed that the threat will prefer the COA that will offer to the adversary the greatest advantages while minimizing risk. Table 4 presents the intelligence matrix as completed by the analyst. The used linguistic scale for evaluation is as follows: VL, very low; L, low; M, medium; $\mathrm{H}$, high; and $\mathrm{VH}$, very high.

4.2. Step 2: Handling Imperfect Data. In this situation, the analyst makes the choice of triangular fuzzy number to model the linguistic variables. The used membership function is given by (2) (see Section 3.3), where parameters of the membership functions $\left(a_{1}, a_{2}, a_{3}\right)$ are given in Table 5 .

4.3. Step 3: Modeling the Analyst's Risk Attitude. When evaluating the different competitor's/threat's actions, the intelligence analyst will express different risk attitudes (from absolutely optimistic to absolutely pessimistic), depending on the context, on the significance of the action, and on its consequences. We assume in this illustration that the attitude towards risk of the intelligence analyst is optimistic. To incorporate the risk attitude to the triangular fuzzy number, the transformation proposed in Fenton and Wang [27] is used (see Table 2). The transformed fuzzy numbers associated with each linguistic term will be transformed using the following formulas: $\left(a_{1},\left(a_{2}+a_{3}\right) / 2, a_{3}\right)$ for criteria to be minimized and $\left(a_{1},\left(a_{2}+a_{1}\right) / 2, a_{3}\right)$ for criteria to be maximized. As a result, 
TABLE 4: Intelligence matrix.

\begin{tabular}{lcccc}
\hline & $A 1$ & $A 2$ & $A 3$ & $A 4$ \\
\hline$C 1(\max )$ & $\mathrm{VH}$ & $\mathrm{H}$ & $\mathrm{VH}$ & $\mathrm{M}$ \\
$C 2(\max )$ & $\mathrm{M}$ & $\mathrm{VH}$ & $\mathrm{H}$ & $\mathrm{VH}$ \\
$C 3(\max )$ & $\mathrm{L}$ & $\mathrm{M}$ & $\mathrm{H}$ & $\mathrm{H}$ \\
$C 4(\max )$ & $\mathrm{VH}$ & $\mathrm{H}$ & $\mathrm{M}$ & $\mathrm{VH}$ \\
$C 5(\max )$ & $\mathrm{H}$ & $\mathrm{M}$ & $\mathrm{VL}$ & $\mathrm{VH}$ \\
$C 6(\min )$ & $\mathrm{H}$ & $\mathrm{M}$ & $\mathrm{VH}$ & $\mathrm{VL}$ \\
$C 7(\min )$ & $\mathrm{H}$ & $\mathrm{H}$ & $\mathrm{M}$ & $\mathrm{L}$ \\
$C 8(\min )$ & $\mathrm{H}$ & $\mathrm{VH}$ & $\mathrm{M}$ & $\mathrm{L}$ \\
$C 9(\max )$ & $\mathrm{M}$ & $\mathrm{M}$ & $\mathrm{H}$ & $\mathrm{VH}$ \\
$C 10(\max )$ & $\mathrm{L}$ & $\mathrm{VL}$ & $\mathrm{M}$ & $\mathrm{M}$ \\
$C 11(\min )$ & $\mathrm{VH}$ & $\mathrm{VH}$ & $\mathrm{M}$ & $\mathrm{L}$ \\
$C 12(\min )$ & $\mathrm{VH}$ & $\mathrm{VH}$ & $\mathrm{L}$ & $\mathrm{M}$ \\
$C 13(\min )$ & $\mathrm{VH}$ & $\mathrm{VH}$ & $\mathrm{H}$ & $\mathrm{H}$ \\
\hline
\end{tabular}

TABle 5: Membership functions.

\begin{tabular}{lc}
\hline Linguistic term & Membership function $\left(a_{1}, a_{2}, a_{3}\right)$ \\
\hline Very low $(\mathrm{VL})$ & $(0.00,0.10,0.25)$ \\
Low $(\mathrm{L})$ & $(0.15,0.30,0.45)$ \\
Medium $(\mathrm{M})$ & $(0.35,0.50,0.65)$ \\
High $(\mathrm{H})$ & $(0.55,0.70,0.85)$ \\
Very high $(\mathrm{VH})$ & $(0.75,0.90,1.00)$ \\
\hline
\end{tabular}

the membership incorporating the analyst's risk attitude will be as shown in Table 6 .

Table 7 gives the intelligence matrix obtained by using the transformation in Table 6.

4.4. Step 4: Multicriteria Performance Aggregation. In this step, the analyst will use a fuzzy MCDM method. As discussed earlier in Section 3.4, a relatively great number of methods do exist. We just need in this step of the MCIA methodology to be cautious and to make an informed choice for the method based on its strengths and weaknesses. For the sake of illustration, we will do the multicriteria performance aggregation using fuzzy TOPSIS.

The intelligence matrix is given in Table 7.

(1) Determine the positive ideal $A^{*}$ and the negative ideal $A^{-}$solutions: we consider $J_{1}$ the set of criteria to be maximized and $J_{2}$ the set of criteria to be minimized and determine the positive ideal and negative ideal using the following formulas:

$$
\begin{aligned}
A^{*} & =\left(v_{1}^{*}, v_{2}^{*}, \ldots, v_{j}^{*}, \ldots, v_{m}^{*}\right)=\left(\max _{i} v_{i j} \mid j\right. \\
& \left.\in J_{1}, \min _{i} v_{i j} \mid j \in J_{2}, i=1, \ldots, N\right), \\
A^{*} & =(\mathrm{VH}, \mathrm{VH}, \mathrm{H}, \mathrm{VH}, \mathrm{VH}, \mathrm{VL}, \mathrm{L}, \mathrm{L}, \mathrm{VH}, \mathrm{M}, \mathrm{L}, \mathrm{L}, \mathrm{H}),
\end{aligned}
$$

TABLE 6: Membership functions with the risk attitude of the analyst.

\begin{tabular}{lcc}
\hline Linguistic term & $\begin{array}{c}\text { Membership function } \\
\text { for criteria to be } \\
\text { minimized }\end{array}$ & $\begin{array}{c}\text { Membership function } \\
\text { for criteria to be } \\
\text { maximized }\end{array}$ \\
\hline Very low (VL) & $(0,0,175,0,25)$ & $(0,0,05,0,25)$ \\
Low (L) & $(0,15,0,375,0,45)$ & $(0,15,0,225,0,45)$ \\
Medium (M) & $(0,35,0,575,0,65)$ & $(0,35,0,425,0,65)$ \\
High (H) & $(0,55,0,775,0,85)$ & $(0,55,0,625,0,85)$ \\
Very high (VH) & $(0,75,0,95,1)$ & $(0,75,0,825,1)$ \\
\hline \multicolumn{3}{c}{$A^{-}=\left(v_{1}^{-}, v_{2}^{-}, \ldots, v_{j}^{-}, \ldots, v_{m}^{-}\right)=\left(\min _{i} v_{i j} \mid j\right.$} \\
$\left.\quad \in J_{1}, \max _{i} v_{i j} \mid j \in J_{2}, i=1, \ldots, N\right)$, \\
$A^{-}=(\mathrm{M}, \mathrm{M}, \mathrm{L}, \mathrm{M}, \mathrm{VL}, \mathrm{VH}, \mathrm{H}, \mathrm{VH}, \mathrm{M}, \mathrm{VL}, \mathrm{VH}, \mathrm{VH}, \mathrm{VH})$.
\end{tabular}

(2) Calculate the separation measures for each alternative $i$ : the separation from the positive ideal and the negative ideal alternative is given by

$$
\begin{aligned}
& S_{i}^{*}=\sum_{j=1}^{n} d\left(v_{i j}-v_{j}^{*}\right), \\
& S_{i}^{-}=\sum_{j=1}^{n} d\left(v_{i j}-v_{j}^{-}\right),
\end{aligned}
$$

where $n$ is the number of criteria.

Consider

$$
d(a, b)=\sqrt{\frac{1}{3\left[\left(a_{1}-b_{1}\right)^{2}+\left(a_{2}-b_{2}\right)^{2}+\left(a_{3}-b_{3}\right)^{2}\right]}}
$$

The results are given in Table 8 .

(3) Calculate the relative closeness to the ideal solution using the following equation:

$$
C_{i}^{*}=\frac{S_{i}^{-}}{S_{i}^{*}+S_{i}^{-}}
$$

The results of the Fuzzy TOPSIS recommends $A 3$ which has the maximum value for $C^{*}$ (see Table 9 ).

With these results, the analyst will be provided with an indication on which action is more likely to be chosen by the threat. As action generation was based on case-based reasoning (in Step 1), the four actions considered here are similar to what the threat had chosen to do in the past. The MCIA analysis of these 4 actions will give the analyst insights on the ranking of the four actions. Considering the list of criteria and the attitude towards risk of the analyst, results show that action $A 3$ is more likely to be chosen, followed by $A 1, A 2$, and $A 4$. Once this information is known, military experts will make informed decisions and therefore get an advantage on the threat. 
TABLE 7: Intelligence matrix.

\begin{tabular}{lcccc}
\hline & $A 1$ & $A 2$ & $A 3$ & $A 4$ \\
\hline$C 1(\max )$ & $(0,75,0,825,1)$ & $(0,55,0,625,0,85)$ & $(0,75,0,825,1)$ & $(0,35,0,425,0,65)$ \\
$C 2(\max )$ & $(0,35,0,425,0,65)$ & $(0,75,0,825,1)$ & $(0,55,0,625,0,85)$ & $(0,75,0,825,1)$ \\
$C 3(\max )$ & $(0,15,0,225,0,45)$ & $(0,35,0,425,0,65)$ & $(0,55,0,625,0,85)$ & $(0,55,0,625,0,85)$ \\
$C 4(\max )$ & $(0,75,0,825,1)$ & $(0,55,0,625,0,85)$ & $(0,35,0,425,0,65)$ & $(0,75,0,825,1)$ \\
$C 5(\max )$ & $(0,55,0,775,0,85)$ & $(0,35,0,425,0,65)$ & $(0,0,05,0,25)$ & $(0,75,0,825,1)$ \\
$C 6(\min )$ & $(0,55,0,775,0,85)$ & $(0,35,0,575,0,65)$ & $(0,75,0,95,1)$ & $(0,0,175,0,25)$ \\
$C 7(\min )$ & $(0,55,0,775,0,85)$ & $(0,55,0,775,0,85)$ & $(0,35,0,575,0,65)$ & $(0,15,0,375,0,45)$ \\
$C 8(\min )$ & $(0,55,0,775,0,85)$ & $(0,75,0,95,1)$ & $(0,35,0,575,0,65)$ & $(0,75,0,825,1)$ \\
$C 9(\max )$ & $(0,35,0,425,0,65)$ & $(0,35,0,425,0,65)$ & $(0,55,0,625,0,85)$ & $(0,35,0,425,0,65)$ \\
$C 10(\max )$ & $(0,15,0,225,0,45)$ & $(0,0,05,0,25)$ & $(0,35,0,425,0,65)$ & $(0,15,0,375,0,45)$ \\
$C 11(\min )$ & $(0,75,0,95,1)$ & $(0,75,0,95,1)$ & $(0,35,0,575,0,65)$ & $(0,35,0,575,0,65)$ \\
$C 12(\min )$ & $(0,75,0,95,1)$ & $(0,75,0,95,1)$ & $(0,15,0,375,0,45)$ & $(0,55,0,775,0,85)$ \\
$C 13(\min )$ & $(0,75,0,95,1)$ & $(0,75,0,95,1)$ & $(0,55,0,775,0,85)$ & \\
\hline
\end{tabular}

TABLE 8: Separation measures.

\begin{tabular}{ccccc}
\hline & $A 1$ & $A 2$ & $A 3$ & $A 4$ \\
\hline$S^{*}$ & 4,2260 & 4,3997 & 2,8279 & 5,8992 \\
$S^{-}$ & 1,9260 & 1,7352 & 2,9572 & 0,5841 \\
\hline
\end{tabular}

TABLE 9

\begin{tabular}{lllll}
\hline$C^{*}$ & 0,3131 & 0,2828 & 0,5112 & 0,0901 \\
\hline
\end{tabular}

As in the military context, the methodology will be valuable in the business context. In fact, it will provide business executives with better informational and intelligence support that provides them with a basis for improved decisionmaking. Better decisions will in turn lead to reaching the established business goals. By having a proactive attitude (anticipating what is going to happen instead of simply reacting), the company will better understand external influences, make timely and right decisions, and then get a better position in the market.

Compared to classical MCDA, the proposed methodology will be part of the intelligence process, which is prior to the decision-making process. It is concerned with the competitor/threat decisions and not with our own decisionmaking process as in MCDA. In fact, MCDA involves the decision-maker in the process; however, MCIA cannot do it. While MCDA uses several techniques to elucidate the decision-maker's preference modeling (as direct elicitation, AHP, etc.), MCIA uses reasoning techniques from artificial intelligence to infer information on the competitor/threat. Furthermore, MCIA cannot evolve other than in a context of uncertainty, incompleteness, and ambiguity because of partial ignorance on the competitor/threat. This increases the complexity and the challenges of the problem. The new MCIA field is promising and needs further research in order to continue developing this methodology. For instance, development in the area of competitor threat preference modeling is required.

\section{Conclusion}

This paper extends the multicriteria decision aid methodology to the context of intelligence analysis. The proposed "Multicriteria Intelligence Aid" (MCIA) methodology aims at supporting the intelligence analyst in structuring the competitor/threat decision problem and in anticipating their actions. Its main pillars are exposed in the paper. The methodology uses a panoply of techniques from artificial intelligence, MCDA, and fuzzy multicriteria decision analysis and shows how existent techniques in these areas could be combined together in order to develop such a methodology. The methodology was illustrated with an application in the military context. Results show that the novel methodology is applicable and provides interesting and valuable results for the analyst. As intelligence is increasingly relevant today in a big data context, this methodology will support business executives, military, and governments in exploiting the huge amount of data and anticipating threat/competitor future decisions.

\section{Conflicts of Interest}

The author declares that there are no conflicts of interest regarding the publication of this paper.

\section{References}

[1] H. Abbass, A. Bender, S. Gaidow, and P. Whitbread, "Computational red teaming: Past, present and future," IEEE Computational Intelligence Magazine, vol. 6, no. 1, pp. 30-42, 2011.

[2] K. P. Coyne and J. Horne, Predicting your competitors reactions, Harvard Business School, publishing corporation, 2009.

[3] H. A. Abbass, "An economical cognitive approach for biobjective optimization using bliss points, visualization, and interaction," Soft Computing, vol. 10, no. 8, pp. 687-698, 2006.

[4] K. C. Tan, Y. J. Yang, and C. K. Goh, "A distributed cooperative coevolutionary algorithm for multiobjective optimization," IEEE Transactions on Evolutionary Computation, vol. 10, no. 5, pp. 527-549, 2006. 
[5] M. T. Jensen, "Helper-objectives: using multi-objective evolutionary algorithms for single-objective optimisation," Journal of Mathematical Modelling and Algorithms, vol. 1, no. 25, 2004.

[6] C. A. C. Coello, D. A. V. Van Veldhuizen, and G. B. Lamont, Evolutionary algorithms for solving multi-objective problems, vol. 5 of Genetic Algorithms and Evolutionary Computation, Kluwer Academic, New York, NY, USA, 2002.

[7] H. A. Abbass, R. Sarker, and C. Newton, "PDE: A pareto frontier differential evolution approach for multi-objective optimization problems," in Proceedings of the Evolutionary Computation, vol. 2, pp. 971-978, IEEE Service Centre, Seoul, South Korea.

[8] K. Miettinen, Nonlinear Multiobjective Optimization, Kluwer Academic Publishers, Norwell, Mass, USA, 1999.

[9] B. Roy, "Méthodologie multicritère d'aide à la decision," Economica, 1985, Paris.

[10] N. Fenton and M. Neil, "Making decisions: Using Bayesian nets and MCDA," Knowledge-Based Systems, vol. 14, no. 7, pp. 307325, 2001.

[11] W. Watthayu and Y. Peng, "A Bayesian network based framework for multi-criteria decision making," in $M C D M$, pp. 6-11, B.C. Canada, Whistler, 2004.

[12] R. E. Bellman and L. A. Zadeh, "Decision-making in a fuzzy environment," Management Science, vol. 17, pp. 141-164, 1970.

[13] C. Robert and R. Fuller, "Fuzzy multiple criteria decisionmaking: recent developments," Fuzzy Sets and Systems, vol. 78, pp. 139-153, 1996.

[14] R. A. Ribeiro, "Fuzzy multiple attribute decision making: a review and new preference elicitation techniques," Fuzzy Sets and Systems, vol. 78, no. 2, pp. 155-181, 1996.

[15] R. R. Yager, "On the valuation of alternatives for decisionmaking under uncertainty," International Journal of Intelligent Systems, vol. 17, no. 7, pp. 687-707, 2002.

[16] R. R. Yager, "Fuzzy modeling for intelligent decision making under uncertainty," IEEE Transactions on Systems, Man, and Cybernetics, Part B: Cybernetics, vol. 30, no. 1, pp. 60-70, 2000.

[17] C. T. Chen, "Extensions of the TOPSIS for group decisionmaking under fuzzy environment," Fuzzy Sets and Systems, vol. 114, no. 1, pp. 1-9, 2000.

[18] H. Deng, "Multicriteria analysis with fuzzy pairwise comparison," International Journal of Approximate Reasoning, vol. 21, no. 3, pp. 215-231, 1999.

[19] C. H. Cheng, K. L. Yang, and C. L. Hwang, "Evaluating attack helicopters by AHP based on linguistic variable weight," European Journal of Operational Research, vol. 116, no. 2, pp. 423-435, 1999.

[20] C. H. Yeh and H. Deng, "An algorithm for fuzzy multi-criteria decision making," in Proceeding of the IEEE International Conference on Intelligent Processing Systems, pp. 1564-1568, Beijing, China, 1997.

[21] R. R. Yager, "Multiple objective decision-making using fuzzy sets," International Journal of Man-Machine Studies, vol. 9, no. 4, pp. 375-382, 1977.

[22] S. M. Baas and H. Kwakernaak, "Rating and ranking of multipleaspect alternatives using fuzzy sets," Automatica, vol. 13, no. 1, pp. 47-58, 1977.

[23] D. Dubois and H. Prade, "The use of fuzzy numbers in decision analysis," in Fuzzy information and decision processes, pp. 309321, North-Holland, Amsterdam-New York, 1982.

[24] T. Y. Tseng and C. M. Klein, "A new algorithm for fuzzy multicriteria decision making," International Journal of Approximate Reasoning, vol. 6, no. 1, pp. 45-66, 1992.
[25] L. N. Jakubchak, The Effectiveness of Multi-Criteria Intelligence Matrices in Intelligence Analysis, Master of Science in Applied Intelligence Mercyhurst College [Master, thesis], 2009.

[26] R. Keeney, Value Focused Thinking, Harvard University Press, 1992.

[27] N. E. Fenton and W. Wang, "Risk and confidence analysis for fuzzy multicriteria decision making," Knowledge-Based Systems, vol. 19, no. 6, pp. 381-446, 2006.

[28] T. H. Stewart, "Dealing with uncertainties in MCDA," in International Series in Operations Research and Management Science, F. Jose, G. Salvatore, and E. Matthias, Eds., vol. 78, pp. 445-466, Springer, New York, NY, USA.

[29] R. M. Tong and P. P. Bonissone, "A linguistic approach to decision making with fuzzy sets," Institute of Electrical and Electronic Engineers Transactions on systems, man and cybernetics, vol. 10, no. 11, pp. 716-723, 1980.

[30] F. Herrera and E. Herrera-Viedma, "Linguistic decision analysis: steps for solving decision problems under linguistic information," Fuzzy Sets and Systems, vol. 115, no. 1, pp. 67-82, 2000.

[31] F. Herrera, E. Herrera-Viedma, and L. Martínez, "A fusion approach for managing multi-granularity linguistic term sets in decision making," Fuzzy Sets and Systems, vol. 114, no. 1, pp. 4358, 2000.

[32] G. A. Miller, "The magical number seven, plus or minus two: some limits on our capacity for processing information," The Psychological Review, vol. 63, no. 2, pp. 81-97, 1956. 


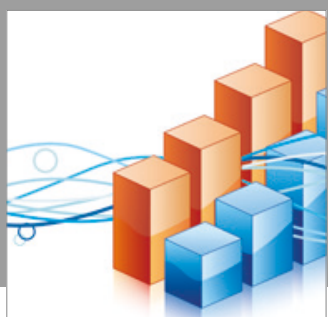

Advances in

Operations Research

vatersals

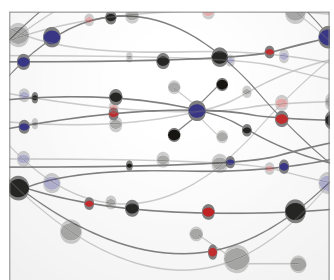

\section{The Scientific} World Journal
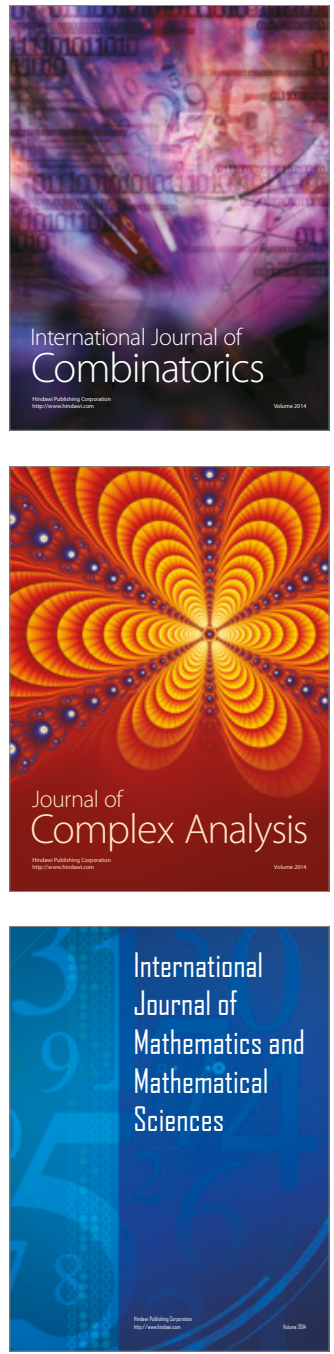
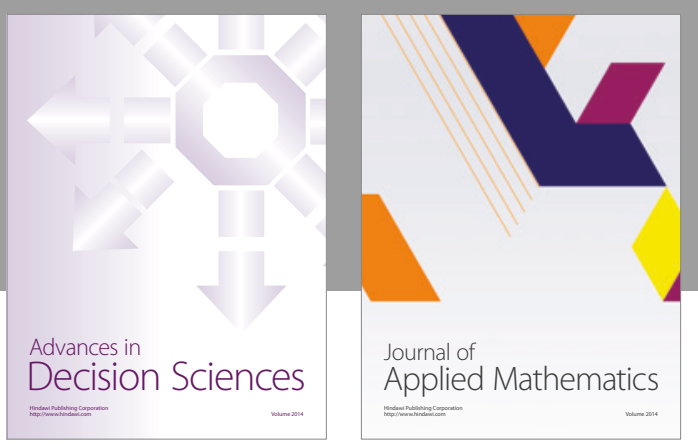

Algebra

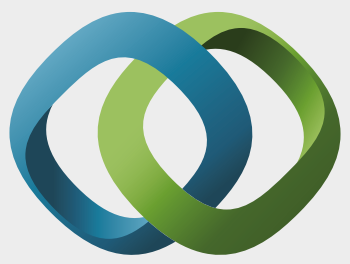

\section{Hindawi}

Submit your manuscripts at

https://www.hindawi.com
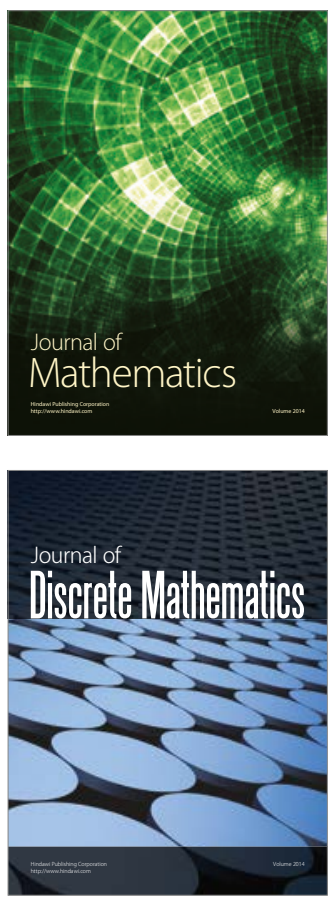

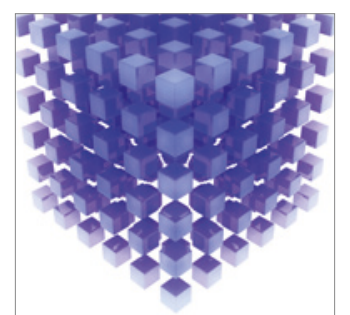

Mathematical Problems in Engineering
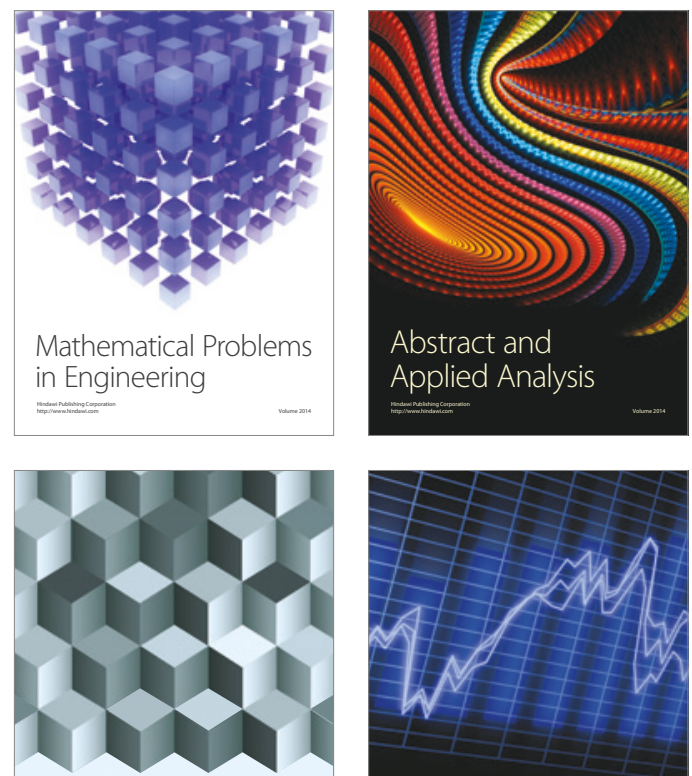

Journal of

Function Spaces

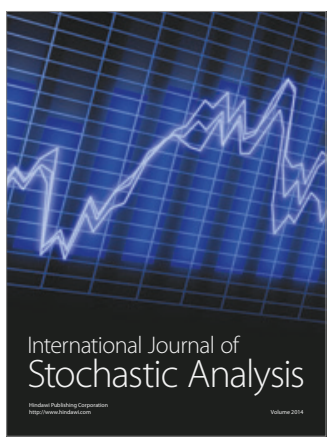

Probability and Statistics
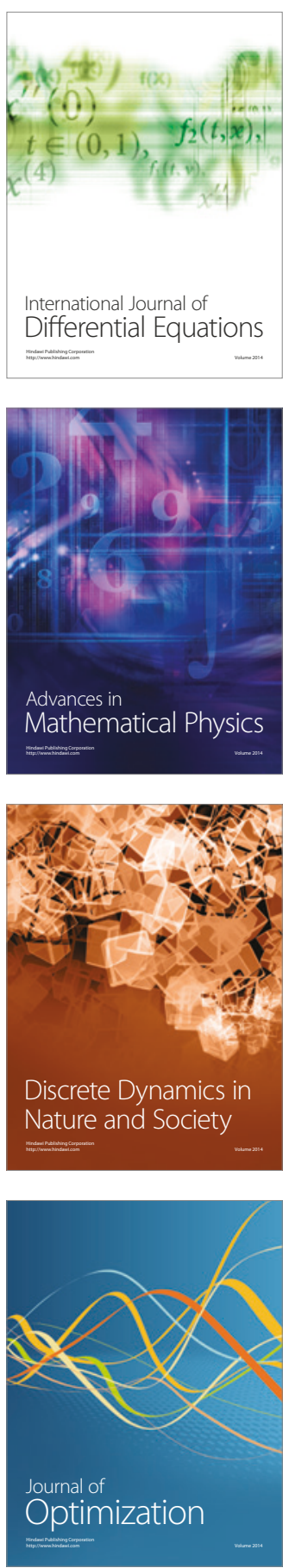